\title{
Editorial
}

\section{The Phoenix Arises Again: The 8th-year Celebration of the Journal of Epidemiology and Global Health (JEGH)}

The Journal of Epidemiology and Global Health (JEGH) celebrates its 8th year of publication since it was first launched in November 2011. JEGH was the first official journal of the Saudi Arabian Ministry of Health, with Elsevier as the publishing services provider till Atlantis Press acquired it at the end of 2018.

The Journal of Epidemiology and Global Health is an International Open Access, peer-reviewed journal focused on innovative scholarship and strategies to advance global health. This quarterly published journal is already an indispensable resource for public health professionals, epidemiologists, and infectious disease specialists.

From the outset, the aim of JEGH was to become the foremost scientific, peer-reviewed international journal encompassing the diverse yet overlapping areas of infectious diseases, microbiology, public health, and global health. JEGH continues to be especially focused on the application of healthcare epidemiology science to these areas and, in doing so, commits to foster a growing body of literature dedicated to the evaluation of health outcomes processes. The truly international editorial board of JEGH launches these efforts with the abiding belief that epidemiology and global health remain closely intertwined, perhaps never more so than now in the flat, globalized world where advances in one area rapidly affect the others.

At JEGH, we aim to improve global healthcare delivery at a global level by reducing the risk of infectious diseases with focus on the rising rates of noncommunicable diseases and related adverse outcomes through critical review, selection, and dissemination of new and relevant information. We seek to disperse this knowledge in all healthcare settings and communities, both regionally and internationally.

The Journal of Epidemiology and Global Health cultivates opportunities to collaborate with others, who share such aims no matter their provenance. Through this intellectual exchange, we seek to enhance our work to uphold the standards of our profession while contributing to its international advancement.

Since 2011, the journal editors and editorial board worked hard to ensure the journal was indexed in a record time of 18 months after the journal was launched in Medline (PubMed), Scopus, Emerging Source Citation Index, Directory of Open Access Journals, and other databases.

The secrets behind the success of JEGH and rapid attainment of indexing in the national library of medicine in the record short time were the following key points:

1) Inclusion of international editors covering the six $\mathrm{WHO}$ regions lends the journal an international authority and extends its readership beyond the Middle East while reflecting the natural history of modern healthcare in the Arabian Gulf. An international leadership facilitates the publication of papers that reflect a new global view of public health and epidemiology while emphasizing our focus on supporting the needs of public health practitioners and epidemiologists across the globe.

2) The Journal of Epidemiology and Global Health acts as a tipping point to change the regional medical culture. Most significantly, we aim to ignite dialogue between subspecialties in the Middle East region as it prepares for an unprecedented growth in all aspects of the healthcare sector. The ability to critically assess scientific literature and distill its value to the bedside is a key feature of providing excellent healthcare important for any clinician to master. JEGH seeks to stimulate these processes in the Middle East Arab region, where the culture of interacting with the medical literature whether as an author, reviewer, editor, and/or critic remains young and in evolution.

3) Furthermore, a journal lead from the region also raises the bar for regional academicians who will now be more willing to access opportunities to publish perhaps by emulating their local colleagues who are leading this effort. The practice of publishing and submitting rather than being a remote, impossible-to-attain fantasy may become more tenable as a realistic professional goal. We wish to inspire this shift in cultural attitudes to medical publishing by providing a forum in which local clinicians may wish to contribute while holding them to the highest international standards of scientific publication.

4) The Journal of Epidemiology and Global Health is a vehicle for the emerging discipline of Mass Gathering Medicine, which is a growing area of concern for emerging infectious diseases spread and the need to respond rapidly to potential and actual outbreaks is intense and increasingly well documented.

The journal has both international leadership and global relevance; served by a large and highly qualified editorial board, its members represent the extremely broad range of interests characterizing JEGH itself to the extent possible.

The Journal of Epidemiology and Global Health demonstrates significant content in core biomedical topics. We publish content that is designated "indexable," including research articles, reviews, and in-depth case reports. The geographical breakdown of authorship, downloads, and coverage show that the journal is also performing the key role of enhancing dissemination of research conducted, in particular, in the Middle East and all over the globe. The former needs to be encouraged to advance national and regional academic and scientific missions. 
The Journal of Epidemiology and Global Health is committed to the critical review, careful selection, and prompt publication of new information relevant to the ever-widening fields of epidemiology, public health, and infectious diseases. JEGH fosters opportunities to work with those who share these aims, enhancing such work through mutual partnership. Listing JEGH on Medline and various indexation indices augments its influence and reach, facilitating our vision of sharing expertise more widely.

And to sum it all, JEGH is a journal developed in such an environment that steps forward to communicate matters on increased

Shalimar Shadeed,

Senior Publisher, JEGH, Atlantis Press

Prof. Ziad A. Memish, MD, FRCPC, FRCPE, FACP, FPH

Editor-in-Chief

Correspondence: zmemish@yahoo.com transmission of communicable and noncommunicable diseases and the urgent need for global recognition, awareness, and rapid response to such situations. In the process of disseminating this knowledge, JEGH seeks to change the cultural climate of medical publishing in the Middle East region as well as promote international interactions in the spirit of global health diplomacy. We humbly believe we make a valuable and unique contribution that will remain fresh and revelatory for the reasons detailed hereinbefore. We also sense that collaborative opportunities for those outside the Middle East will be uniquely appealing because of such diverse roles and challenges. 